\title{
Deep Space Acquisition and Tracking with Single Photon Detector Arrays
}

\author{
William Farr*, Suzana Sburlan*, Adit Sahasrabudhe*, and Kevin M. Birnbaum* \\ * Jet Propulsion Laboratory \\ 4800 Oak Grove Drive \\ Pasadena, CA 91109
}

\begin{abstract}
The laser beacon power required by a communication terminal for acquisition and tracking in deep space optical link scenarios can be reduced by a factor of 10 to 100 by replacing an integrating array, such as a CCD, with an array of single photon detectors. An additional benefit of the single photon detector array is that each pixel can have $\mathrm{MHz}$ bandwidths, allowing simultaneous recovery of photon time-ofarrival information that can be used for uplink data recovery or range measurements.
\end{abstract}

\section{INTRODUCTION}

Free space optical communications links from deep space are projected to fulfill future NASA communication requirements for 2020 and beyond [1]. Accurate laser-beam pointing is required to achieve high data rates at low power levels. In order to achieve high pointing accuracy, a laser beacon transmitted from the Earth is acquired and tracked by the space transceiver to obtain accurate knowledge of the Earth receiver position in the pitch and yaw degrees of freedom [2]. The space transceiver focuses the dim beacon onto a focal plane array and estimates the centroid of the resulting spot in order to generate this pointing knowledge. While the focal plane array may be a two by two "quad" detector, a larger array facilitates the initial spatial acquisition [3]. The accuracy of the centroid estimates, and therefore the accuracy of the space transceiver pointing, is a function of the received optical signal power, accepted optical background power, and detector readout noise.

The maximum integration time in forming the estimate is limited by the platform disturbances. In order to control the pointing, the space transceiver must obtain pointing knowledge faster than the disturbances degrade it. Within this integration time, however, there is a limited number of beacon photons available to the transceiver, determined by the beacon laser power. Simple scaling of communication systems designed for near-Earth operating domains (LEO, GEO, lunar, L1-L2) is typically impractical, requiring megawatt powers for deep space distances (due to the much higher $1 / R^{2}$ losses). New technology in spacecraft disturbance rejection systems resolves this problem by enabling second-long integration times to achieve sub-microradian pointing knowledge [4].

Pointing estimates are further degraded by optical background. The sun-illuminated Earth and moon are bright sources of background light. Scattered transmit laser light and scattered solar radiation from near sun pointing operations may

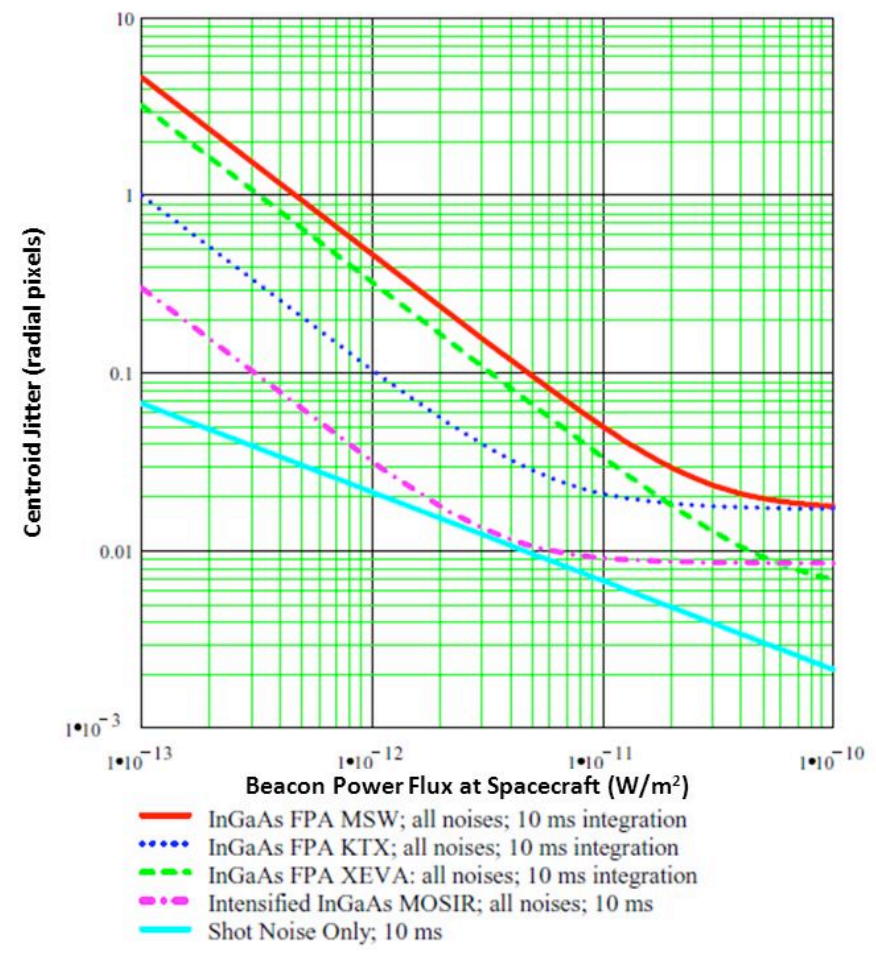

Fig. 1. InGaAs camera centroiding performance.

also contribute, but scattered light contributions are minimized by careful optical system design [5].

The final significant limitation on estimation accuracy is detector noise. As shown in Fig. 1, the centroiding performance of a focal plane array (InGaAs cameras in this example) can be 10 to 100 times poorer than the shot noise limit due to readout noise, although an intensified camera (MOSIR) can nearly achieve shot noise limited performance over a narrow signal range. A focal plane array of single photon detectors can fully close this gap, and thereby require 10 to 100 times less beacon transmit power [6].

\section{ARCHITECTURE}

In addition to forming an accurate estimate of the location of the dim laser beacon, the space transceiver must point its outgoing transmit beam to the Earth receiver location. The space transceiver must therefore accurately control the outgoing 


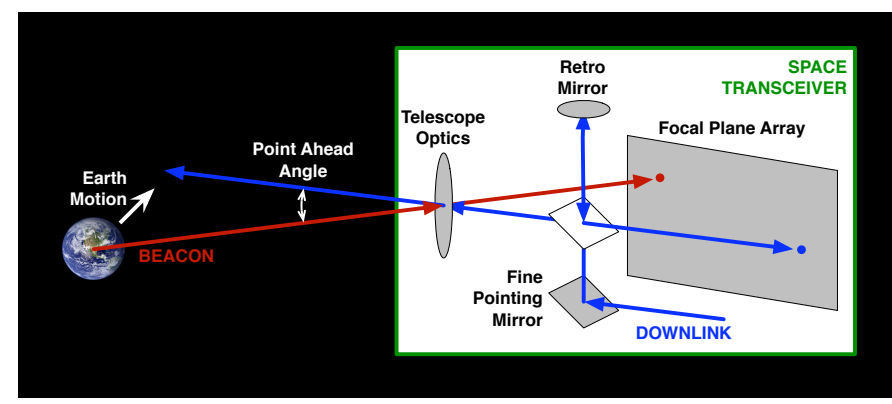

Fig. 2. Space transceiver beam relationships.

beam relative to the beacon. The required angular separation between the beams (the point ahead angle) depends on the distance between the beacon transmitter and the Earth receiver locations and on the transverse component of the Earth's velocity relative to the spacecraft. In deep space applications, the transverse velocity effect can dominate; the spacecraft receives light from where the beacon transmitter was one light propagation time ago, and must point the downlink to where the Earth receiver will be one light propagation time later, which may be many minutes.

A simplified architecture using a single detector to track both the received beacon and transmit laser beams has been previously proposed to reduce the complexity and minimize alignment errors, and this architecture is the basis for the Flight Laser Transceiver subsystem of the proposed DeepSpace Optical Terminals (DOT) project [7].

A cartoon of the pointing subsystem functional components is depicted in Fig. 2. The received Earth laser beacon is imaged onto the receiver focal plane array by the telescope optics, which maps incident angle into a spatial position. A sample of the downlink transmit laser beam is also imaged onto the same focal plane array.

A more detailed depiction of the focal plane image is given in Fig. 3. Here we assume that the received beacon spot has been steered to a predetermined location on the focal plane array by pointing the space transceiver. Enough room is left around this region of the focal plane array to image the sunilluminated Earth. The Earth centroid can then be used to form pointing estimates temporarily if the uplink is lost, allowing the pointing system to "flywheel" through brief uplink outages.

A fixed offset angle is induced by the retro mirror, so that when the downlink beam is pointing in the same direction as the beacon, they do not overlap on the focal plane array. The fine pointing mirror sets the point ahead vector relative to the offset position on the focal plane. The magnitude of the point ahead vector depends on the transverse velocity and the relative positions of the beacon and Earth receive stations. The direction of the point ahead vector (the clock angle) depends on the roll angle of the spacecraft about the line connecting the spacecraft to Earth, which may vary. The point ahead vector will vary over the mission, but the offset angle can be chosen so that the downlink light is never on top of the Earth image.

Each pixel of the focal plane array in Fig. 3 is implemented

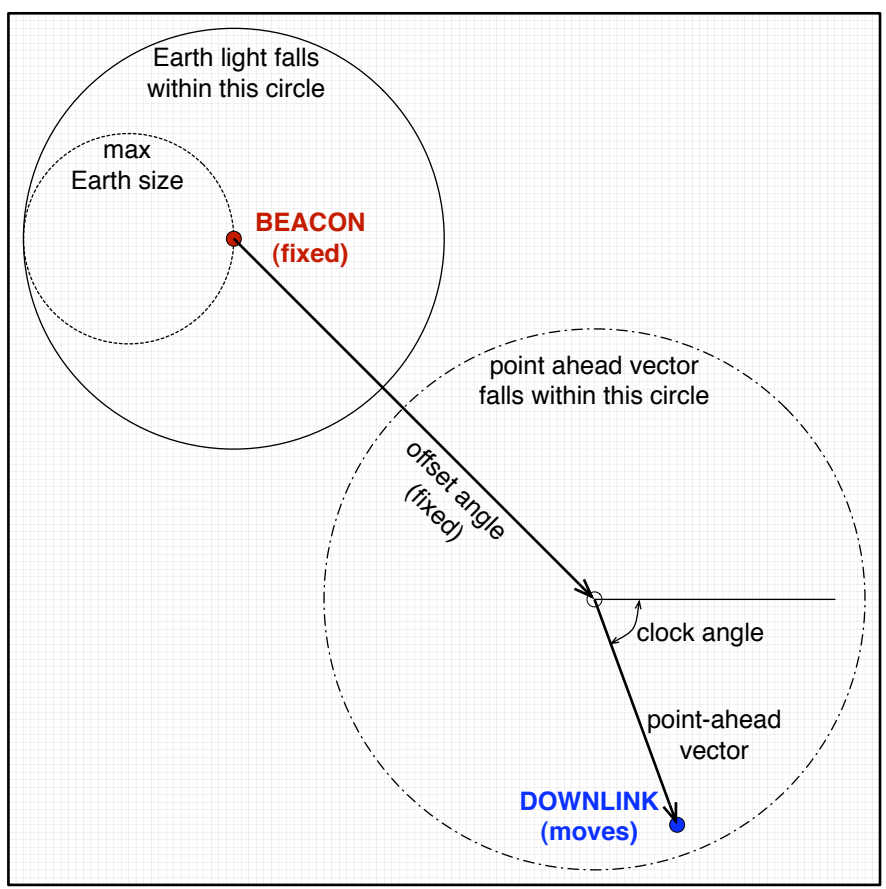

Fig. 3. Space transceiver focal plane image.

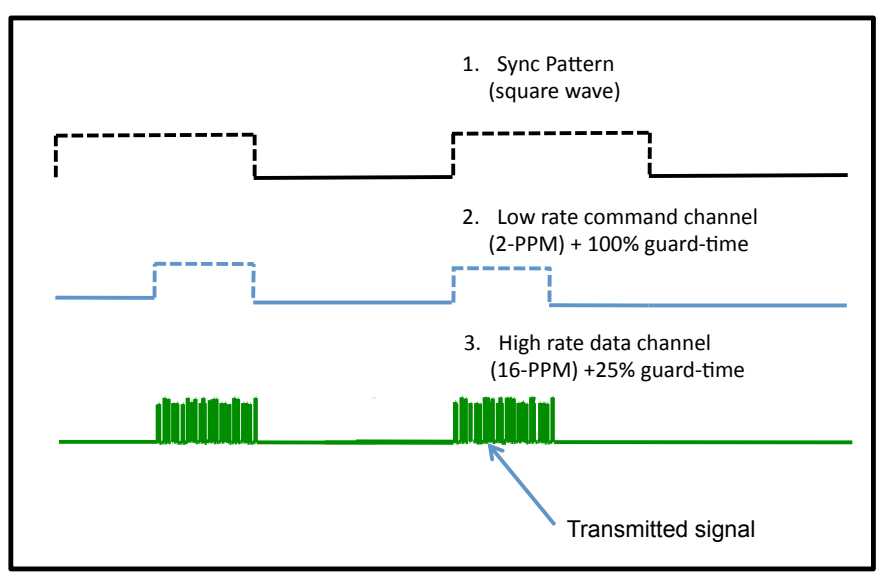

Fig. 4. DOT nested modulation scheme (power vs. time).

with a single photon sensitive detector that ideally outputs a countable electrical pulse for every incident photon. An example of this detector class is the Negative Avalanche Feedback Detector [8]. Using a small $(6 \times 6)$ photon counting focal plane array, we have demonstrated shot noise limited performance in centroid estimation [9].

The laser beacon can be modulated to allow background subtraction of scattered light and upwelling radiance from Earth. The DOT project chose a nested modulation scheme composed of a fixed data rate 2-PPM (pulse-position modulation) outer code with two inter-symbol guard time slots and a variable data rate 16-PPM inner code with four inter-symbol guard time slots [5], [10]. The effect of the nested modulation scheme is to create a square wave sync pattern for "lockin" detection of the laser beacon. A diagram of the signaling 


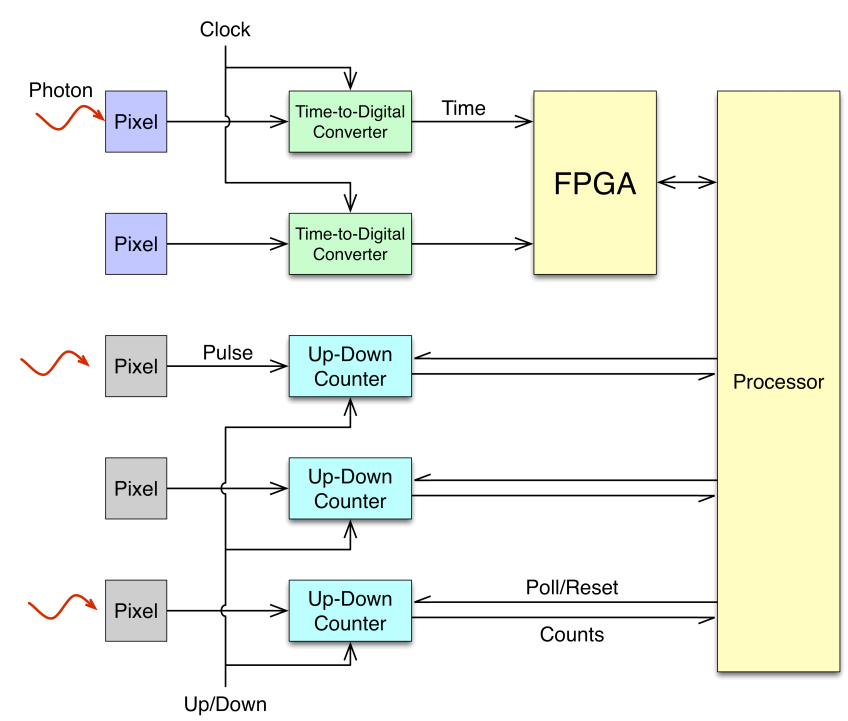

Fig. 5. Fast/slow pixel processing.

structure, showing the resulting power as a function of time, is shown in Fig. 4.

To detect the nested modulation, the space transceiver focal plane array is composed of "slow" and "fast" pixels. A 2x2 or larger sub-array of fast pixels is located at the laser beacon tracking position ("Beacon" in Fig. 3). These pixels record the arrival time of each detected photon relative to an onboard clock for the purpose of data demodulation, as depicted in Fig. 5. This is equivalent to a fast sampled system with sparse vector representation to reduce storage and processing requirements. A field-programmable gate array (FPGA) can be used to reduce demands on the space transceiver baseband processor. For example, the FPGA can be used to perform synchronization and despreading of high rate data.

The remainder of the array consists of the slow pixels. Each slow pixel has an integrating counter under microprocessor control to implement an imaging detector with variable integration time per pixel. During acquisition, the laser beacon spot can be anywhere on the focal plane array, or not present at all. The spacecraft transceiver must distinguish signal from background on the low complexity slow pixels. If the laser beacon spot is not detected, a search scan can be implemented by the space transceiver. During tracking, the space transceiver is pointed to keep the uplink laser beacon spot on to the fast pixels to recover centroid estimates, uplink data, and time-of-flight range.

Spatial acquisition and recovery of the outer modulation data on the uplink laser beacon from Earth can be achieved on the space transceiver focal plane array by connecting two counters to each array pixel. This scheme provides a low complexity method to monitor all pixels in the detector array until a beacon signal is detected. Temporal acquisition of the uplink laser beacon square wave signal is performed using outputs from a pair of phase-offset counters. The counters

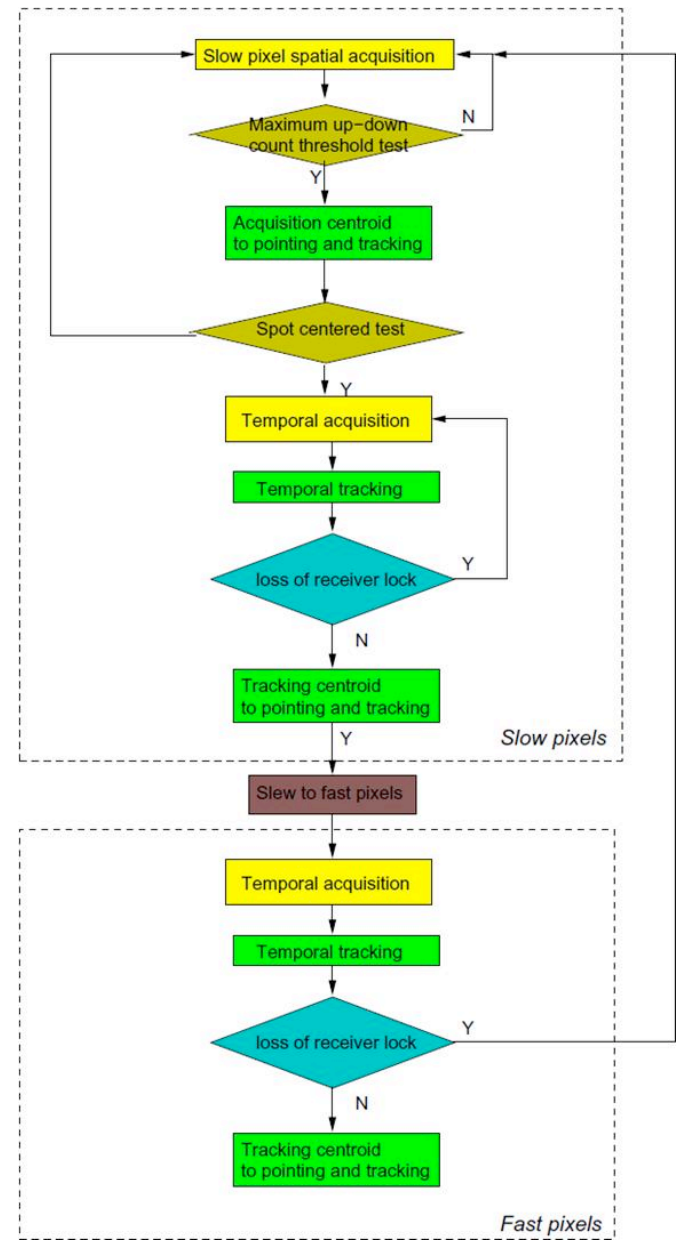

Fig. 6. Acquisition/tracking flowchart.

alternate between three states denoted by "up", "down", and "idle". In the "up" state, a counter increments its value when its pixel registers a photon arrival. In the "down" state the counter decrements its value when a photon arrival is detected. In the "idle" state, the counter maintains its value. For an outer modulation signal of 2 PPM +2 inner-symbol guard time slots, with slot width $T_{\text {slot }}$, the counters cycle through the states with a period of $4 T_{\text {slot }}$. The counters can be viewed as approximations to a maximum-likelihood timing estimation with a modified pulse shape [11]. The counter state can be read and reset by the processor, and estimation based on the counter outputs is done in software.

Combining the two counters yields an estimate of the incident signal level, while allowing the pulses from noise and background radiation to cancel out. Thus, this scheme provides an efficient manner of determining when a signal is present on the array, and to estimate the signal and background levels, which will vary from pixel to pixel. Once a signal is detected at a position in the array, the spacecraft transceiver can be commanded to adjust its pointing to place the laser beacon on the high bandwidth fast pixels, and synchronization to the high data rate inner modulated signal can be carried out. Fig. 6 is 


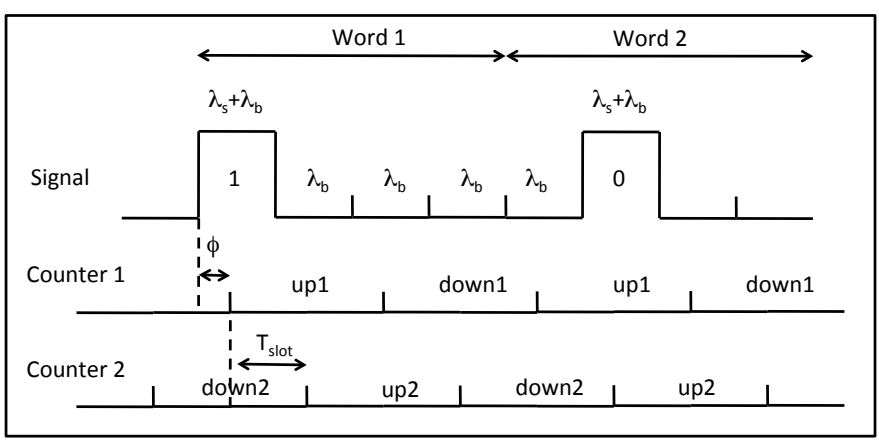

Fig. 7. Timing diagram of signal relative to counters.

a flowchart of the acquisition and tracking process across the slow and fast pixels.

\section{EXAMPLES}

Fig. 7 illustrates the arbitrary alignment of the counters with respect to an incoming signal. The quantities $\lambda_{s}$ and $\lambda_{b}$ refer to the Poisson distributed signal and background count rates respectively. The average number of photons arriving in an interval $t$ is $\left(\lambda_{s}+\lambda_{b}\right) t$. The total time per word is $4 T_{\text {slot }}$. In this example, the counters alternate between the "up" and "down" states every $2 T_{\text {slot }}$. Without being synchronized to the signal, the counters will have a random temporal offset $\phi$. In addition, the two counters for each pixel are out of phase with each other by one slot.

The expectation values for each counters "up" and "down" modes over a period of two words are,

$$
\left.\begin{array}{rl}
\langle\text { up }\rangle & =4 \lambda_{b} T_{\text {slot }}-\lambda_{s} \phi+2 \lambda_{s} T_{\text {slot }} \\
\langle\text { down }\rangle & =4 \lambda_{b} T_{\text {slot }}+\lambda_{s} \phi \\
\langle\text { up }\rangle-\langle\text { down }\rangle & =2 \lambda_{s} T_{\text {slot }}-2 \lambda_{s} \phi \\
\langle\text { up }\rangle+\langle\text { down }\rangle & =8 \lambda_{b} T_{\text {slot }}+2 \lambda_{s} \phi \\
\langle\text { up }\rangle & =4 \lambda_{b} T_{\text {slot }}+\lambda_{s} \phi+\lambda_{s} T_{\text {slot }} \\
\langle\text { down }\rangle & =4 \lambda_{b} T_{\text {slot }}-\lambda_{s} \phi+\lambda_{s} T_{\text {slot }} \\
\langle\text { up }\rangle-\langle\text { down }\rangle & =2 \lambda_{s} \phi \\
\langle\text { up }\rangle+\langle\text { down }\rangle & =8 \lambda_{b} T_{\text {slot }}+2 \lambda_{s} \phi
\end{array}\right\} \text { Counter } 1
$$

A constant background rate $\lambda_{b}$ is assumed across all slots, which can account for the Earth image, scattered light, and detector dark noise. When the counters are alternating between the "up" and "down" modes, the background rate has no average effect on the counter state. Conversely, if the counters are run in the "up" mode only, the background rates are preserved.

The registered number of signal counts in each counter will depend on the offset $\phi$. Because of this offset, the signal counts will be divided between the two counters. Note that when $\phi=T_{\text {slot }}$ counter 1 will register zero counts on average in "up-down" mode. In this case, information about the signal can only be derived from counter 2 . Since the offset $\phi$ can take on any value, we must implement two counters on each pixel to account for this special case. The equations above can be used to form estimators for $\lambda_{s}, \lambda_{b}$, and $\phi$ on each pixel.

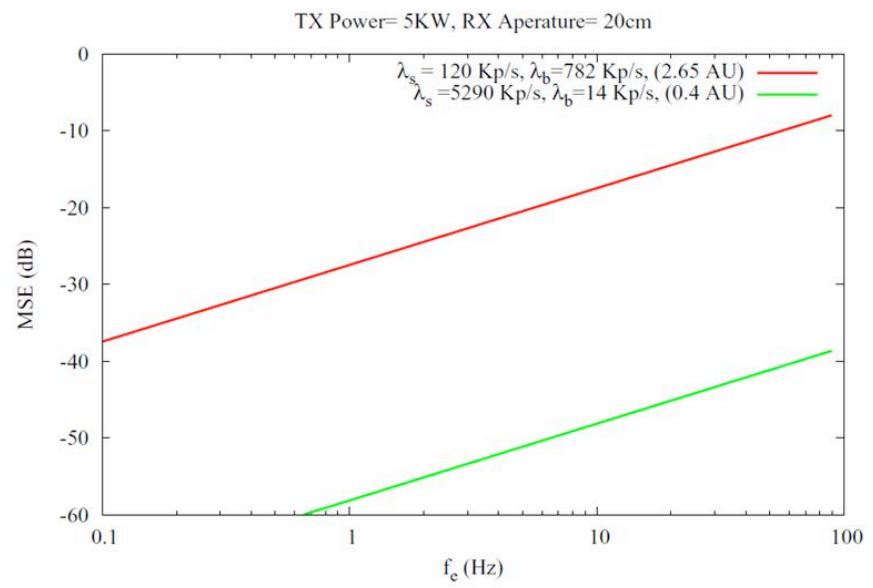

Fig. 8. Mean Square Error of signal estimates vs. estimation rate.

For the estimates of the signal flux to be useful, the relative error on these estimates should be small. This is a function of both the signal and background flux, and the update rate. The higher the update rate the higher the fractional error is. For example, Fig. 8 is a plot of a lower bound on the estimation error for the signal flux estimate at $2.65 \mathrm{AU}$ and $0.4 \mathrm{AU}$ versus the estimation rate, assuming a transmitted beacon power of $5 \mathrm{~kW}$, which is sufficient for the DOT data rate requirements at $0.4 \mathrm{AU}$. As an example in that plot, a relative error on the flux estimate of less than 3\% (less than $-30 \mathrm{~dB}$ Mean Square Error) cannot be achieved at an update rate greater than $1 \mathrm{~Hz}$ at $2.65 \mathrm{AU}$.

\section{CONCLUSION}

Required laser beacon power for acquisition and tracking of a dim beacon in deep space optical link scenarios can be reduced by a factor of 10 to 100 by replacing an analog optical detector array with an array of single photon detectors feeding a set of per-pixel counters. An additional benefit of the single photon detector array is that pixels can have sub-nanosecond timing resolution, allowing simultaneous recovery of photon time-of-arrival information that can be used for uplink data recovery or range measurements. Use of this method allows centroiding performance at the theoretical limit for precision optical beam pointing.

\section{ACKNOWLEDGMENT}

Thanks to Geraldo Ortiz for providing the plots and data in Fig. 1 and Kevin Quirk for the flowchart in Fig. 6 and plot of Fig. 9. The work described here was performed at the Jet Propulsion Laboratory (JPL), California Institute of Technology under contract with the National Aeronautics and Space administration (NASA).

\section{REFERENCES}

[1] (2006, May) NASA Space Communication and Navigation Architecture Recommendations for 2005-2030, Final Report. Space Communications Architecture Working Group. [Online]. Available: https://www.spacecomm.nasa.gov/spacecomm/programs/system planning/default.cfm 
[2] S. Lee, J. W. Alexander, and G. G. Ortiz, "Submicroradian pointing system design for deep-space optical communications," in Proceedings of SPIE, G. S. Mecherle, Ed., vol. 4272, no. 1. SPIE, 2001, pp. 104-111. [Online]. Available: http://dx.doi.org/10.1117/12.430794

[3] C.-C. Chen and J. R. Lesh, "Overview of the optical communications demonstrator," in Proceedings of SPIE, G. S. Mecherle, Ed., vol. 2123, no. 1. SPIE, 1994, pp. 85-94. [Online]. Available: http://dx.doi.org/10.1117/12.184687

[4] V. Sannibale, G. G. Ortiz, and W. H. Farr, "A sub-Hertz vibration isolation platform for a deep space optical communication transceiver," in Proceedings of SPIE, H. Hemmati, Ed., vol. 7199, no. 1. SPIE, 2009, p. 71990I. [Online]. Available: http://dx.doi.org/10.1117/12.810949

[5] A. Biswas, H. Hemmati, S. Piazzolla, B. Moision, K. Birnbaum, and K. Quirk, "Deep-space Optical Terminals (DOT) systems engineering," IPN Progress Reports, vol. 42, no. 183, 2010. [Online]. Available: http://ipnpr.jpl.nasa.gov/progress_report/42-183/183A.pdf

[6] G. G. Ortiz, W. H. Farr, J. R. Charles, W. T. Roberts, V. Sannibale, J. Gin, A. Saharaspude, and V. Garkanian, "Canonical deep space optical communications transceiver," in Proceedings of SPIE, H. Hemmati, Ed., vol. 7199, no. 1. SPIE, 2009, p. 71990K. [Online]. Available: http://dx.doi.org/10.1117/12.812410
[7] W. H. Farr, M. W. Regher, M. W. Wright, A. Sahasrabudhe, J. W Gin, and D. H. Nguyen, "Overview and trades for DOT flight laser transceiver," IPN Progress Reports, vol. 42, no. 184, 2011, (to be published).

[8] W. H. Farr, "Negative avalanche feedback detectors for photon-counting optical communications," in Proceedings of SPIE, H. Hemmati, Ed., vol. 7199, no. 1. SPIE, 2009, p. 71990Q. [Online]. Available: http://dx.doi.org/10.1117/12.814865

[9] S. E. Sburlan, K. M. Birnbaum, and W. H. Farr, "Deep space uplink receiver prototype for optical communications," in Proceedings of SPIE, H. Hemmati, Ed., vol. 7923, no. 1. SPIE, 2011, p. 79230K. [Online]. Available: http://dx.doi.org/10.1117/12.877206

[10] K. Quirk, J. Gin, and M. Srinivasan, "Optical PPM synchronization for photon counting receivers," in IEEE Military Communications Conference, Nov. 2008, pp. 1 -7. [Online]. Available: http://dx.doi.org/ 10.1109/MILCOM.2008.4753054

[11] B. Moision, "Timing from up-down counters," March 2011, JPL internal document. 